\section{Numerical Study of Bach-bladed Savonius Wind Turbine with Varying Blade Shape Factor}

\author{
Kelvin Ibrahim, Vivien S. Djanalif Nur Ikhwan \\ Department of Mechanical Engineering, Institut Teknologi Sepuluh Nopember, Surabaya 60111, Indonesia \\ Received: 6 March 2020, Revised: 5 September 2020, Accepted: 11 September 2020
}

\begin{abstract}
Savonius wind turbine with Bach-profile blades is considered in this study. Previous studies have shown that a rotor with the Bach-profile blade produces better performance than a standard Savonius turbine. This study focuses on the blade shape factor variations of the Bach-profile blade to give the best performance. Two-dimensional unsteady simulations are performed with moving mesh. The configuration being tested is the Savonius rotor with Bach-profile blades with an arc angle of $135^{\circ}$. The blade shape factor is varied $0.2,0.3,0.4$ at a constant freestream velocity of $4 \mathrm{~m} / \mathrm{s}$, with a corresponding Reynolds number of 20,000. The k- $\omega$ Shear Stress Transport turbulence model was used, with secondorder discretization schemes for the pressure and momentum equations. The boundary conditions were set as velocity inlet for the inlet, outflow for the outlet, and walls for the blade surfaces. The top and bottom sides were set as symmetric. Results showed that the configuration with a shape factor of 0.4 gave the best performance among the others. This configuration gave a higher moment coefficient and power coefficient of about $6.8 \%$ and $7.3 \%$, respectively. Results extracted from the simulation includes the flow structure, and the distribution of the pressure coefficients along the blade surface.
\end{abstract}

Keywords: Savonius, Bach, wind turbine, blade shape factor, numerical simulation

\section{Introduction}

The need for energy sources for human survival is very important today. The increasing number of human population and technological advances that support human life result in energy consumption that will increase rapidly. Energy demand in Indonesia will increase from 798 million Barrel of Oil Equivalent in 2015 to 1472 million BOE in 2025. This need is also evidenced by energy consumption, which has increased by $1.3 \%$ per year in the 2010 to 2015 period. Meanwhile, Indonesia's oil reserves continued to decline from 1995 with a value of 5.9 billion barrels to 3.7 billion barrels in 2015 [1].

The problem that occurs due to the increasing energy demand can be solved using renewable energy that can be utilized and developed to replace fossil energy. Renewable energy that can be used and developed is wind energy.

According to data from the Ministry of Energy and Mineral Resources (ESDM), the potential for renewable energy used as energy for power plants reaches $441.7 \mathrm{GW}$ consisting of hydropower $94.3 \mathrm{GW}$, solar power $207.8 \mathrm{GW}$, wind power (wind) $60.6 \mathrm{GW}, 32.6 \mathrm{GW}$ bioenergy, $17.9 \mathrm{GW}$ marine hydropower, and $28.5 \mathrm{GW}$ geothermal. However, the utility of renewable energy is still relatively small compared to its potential. Energy and Mineral Resources Ministry data shows that power plants using renewable energy in 2017 amounted to only $7.3 \mathrm{GW}$ or $1.7 \%$ of the total potential. The utilization of this potential is still very minimal. According to the 2018 EBTKE statistics, the utilization of wind potential in Indonesia is still at $1.96 \mathrm{MW}$. The low utilization of wind energy is caused by the high cost of making horizontal wind turbines and limitations in the placement of this horizontal wind turbine. In Indonesia itself, the relatively low wind speed in the range of $4 \mathrm{~m} / \mathrm{s}$ to $5 \mathrm{~m} / \mathrm{s}$ makes areas in Indonesia need wind turbines with good self-starting capabilities [2].

The Vertical Axis Savonius wind turbine with uncomplicated construction and inexpensive offers a solution to both cost and placement problems. This type of wind turbine construction has several advantages over the horizontal axis, namely that it can absorb wind potential from all directions, works at low speeds, is simple construction and does not require a large installation area, and produces large moments. This is very suitable for the characteristics of the Indonesian wind, which always changes irregularly with relatively small wind speeds. This makes researchers consider choosing a vertical axis wind turbine as an analysis of wind turbine performance in these conditions. Also, the noise generated by the vertical axis wind turbine is smaller than the noise generated by the horizontal axis wind turbine.

The Savonius wind turbine is a vertical wind turbine that has a half-cylinder shape and is installed in the opposite direction. The Savonius wind turbine is a turbine that utilizes the drag force acting on each blade to work. The

*Corresponding author. Email : vivien_s@me.its.ac.id 
difference in drag force from one blade to the other blade will produce torque. This torque can be multiplied by the angular speed of the turbine to obtain turbine power With the greater the difference from this drag force, the power from the turbine will be even greater. Apart from uncomplicated construction and low cost, the Savonius wind turbine has a large starting torque and can run at low speeds. On the other hand, the Savonius wind turbine has a relatively low-efficiency value. Therefore, many studies have been carried out to improve the efficiency of the Savonius wind turbine by modifying the shape of the turbine blades.

Research conducted by Akwa [3] provided a review of the effect of several geometrical parameters on Savonius wind turbines such as end plates, aspect ratio, bucket spacing, and overlap, the number of buckets and rotor levels, buckets and rotor shape, shaft installation, or other accessories, the Reynolds number and turbulence intensity, and the effect of the stator on the performance of the Savonius wind turbine.

Kamoji [4] provided a review of the effect of several geometrical parameters on Savonius wind turbines, such as overlap ratio, aspect ratio, blade arc angle, blade shape factor, and Reynolds number on the performance of the Savonius wind turbine. The results of this study indicated that the optimal coefficient of power of 0.21 was obtained at the Reynolds number value of 150,000 and the arc angle of the Bach turbine $124^{\circ}$.

Other studies [5,6] provided review of the effect of several geometrical parameters on the Savonius wind turbine, such as blade arc angle and blade shape factor, on the performance of the Savonius wind turbine. To study the effect of blade arc angle and blade shape factor on the performance of the twisted blade Savonius hydrokinetic, a three-dimensional, unsteady, and rotating turbine simulation was carried out under this study [5]. Based on the research that has been done, the results showed that the optimum geometric shape parameters for this Savonius Hydrokinetic turbine are the arc angle blade $150^{\circ}$ and blade shape factor 0.6 , and it was found that the increase in the coefficient of power was directly proportional to the increase in velocity flow.

From previous studies, as far as concerns, little research has been carried out on varying the ratio of blade shape factor by simulation method, especially at low speeds. In this research, a comparison of the Bach type Savonius wind turbine will be carried out with varying the blade shape factors of $0.2,0.3$, and 0.4 , at a speed of $4 \mathrm{~m} / \mathrm{s}$ and with a dynamic or rotating turbine condition.

\section{Numerical Method}

This research was conducted by a numerical method using ANSYS FLUENT ${ }^{\complement}$ software. This research using the numerical method in three stages. The stages were the pre-processing, processing, and post-processing stages.

The model made in this study was a two-dimensional geometry of the Bach type Savonius wind turbine and also with a modification to the blade shape factor. At this preprocessing stage, the first process carried out was making the test object geometry. The second process was making meshing on the geometry of the specimen and the simulation domain. The third process was determining the boundary conditions for the simulation. The test object used was a Bach type Savonius wind turbine with a variation of the blade shape factor of $0.2,0.3,0.4$ at a constant wind speed of $4 \mathrm{~m} / \mathrm{s}$, and a Reynolds number of 20,000.

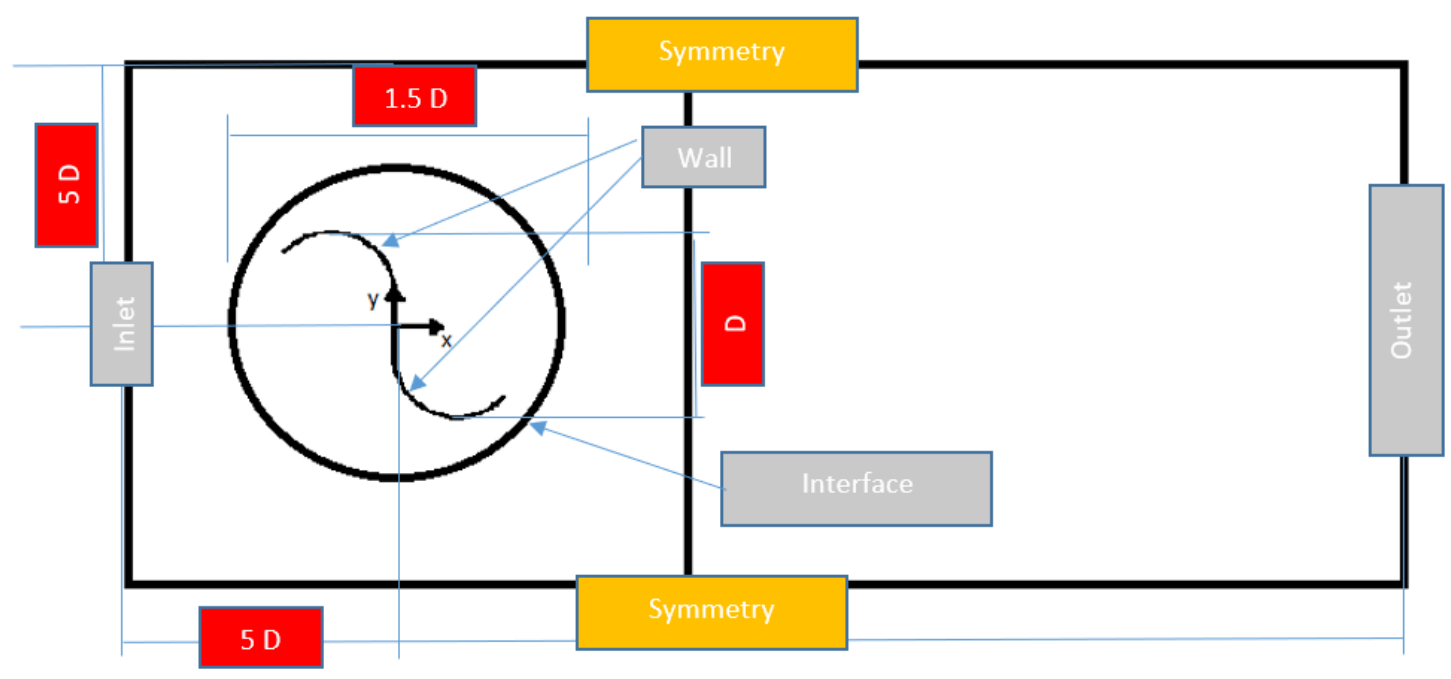

Figure 1. Boundary conditions used in the simulation 


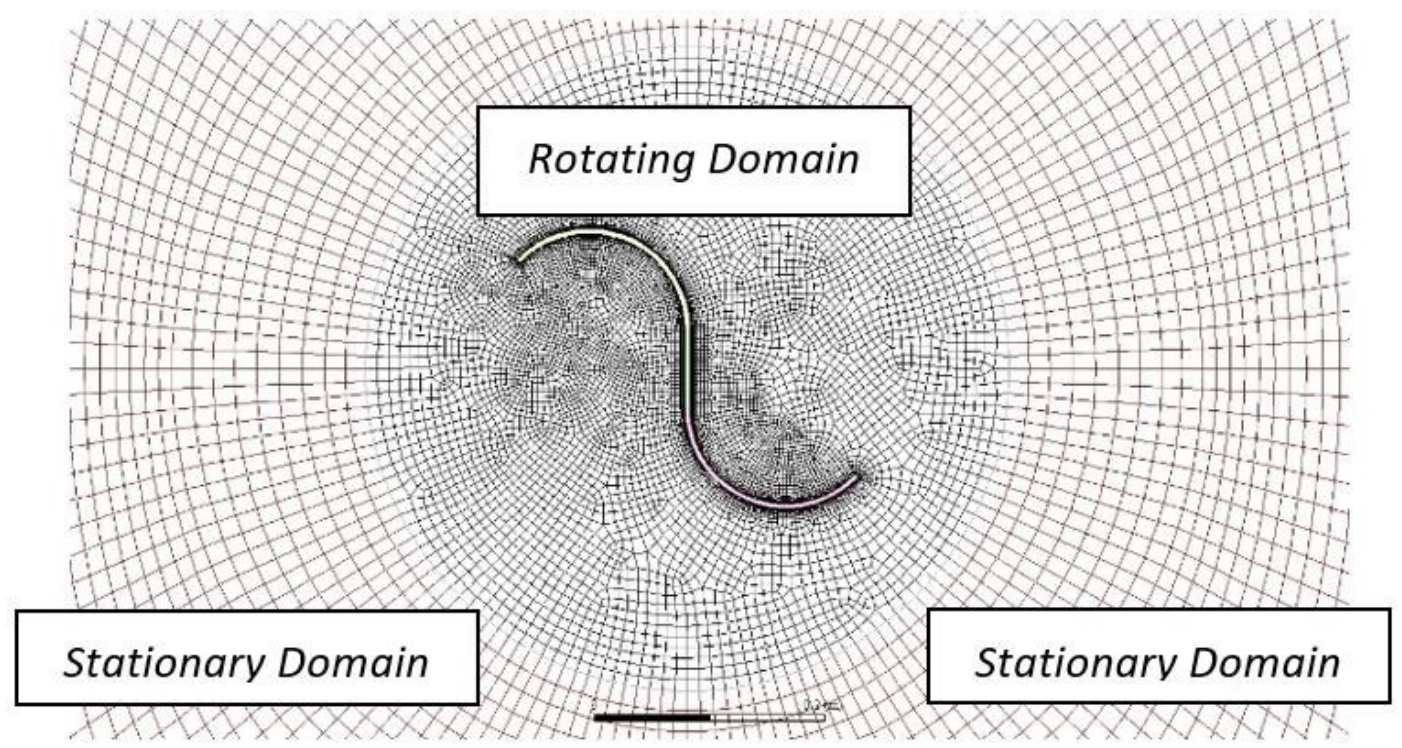

Figure 2. Cell zone condition

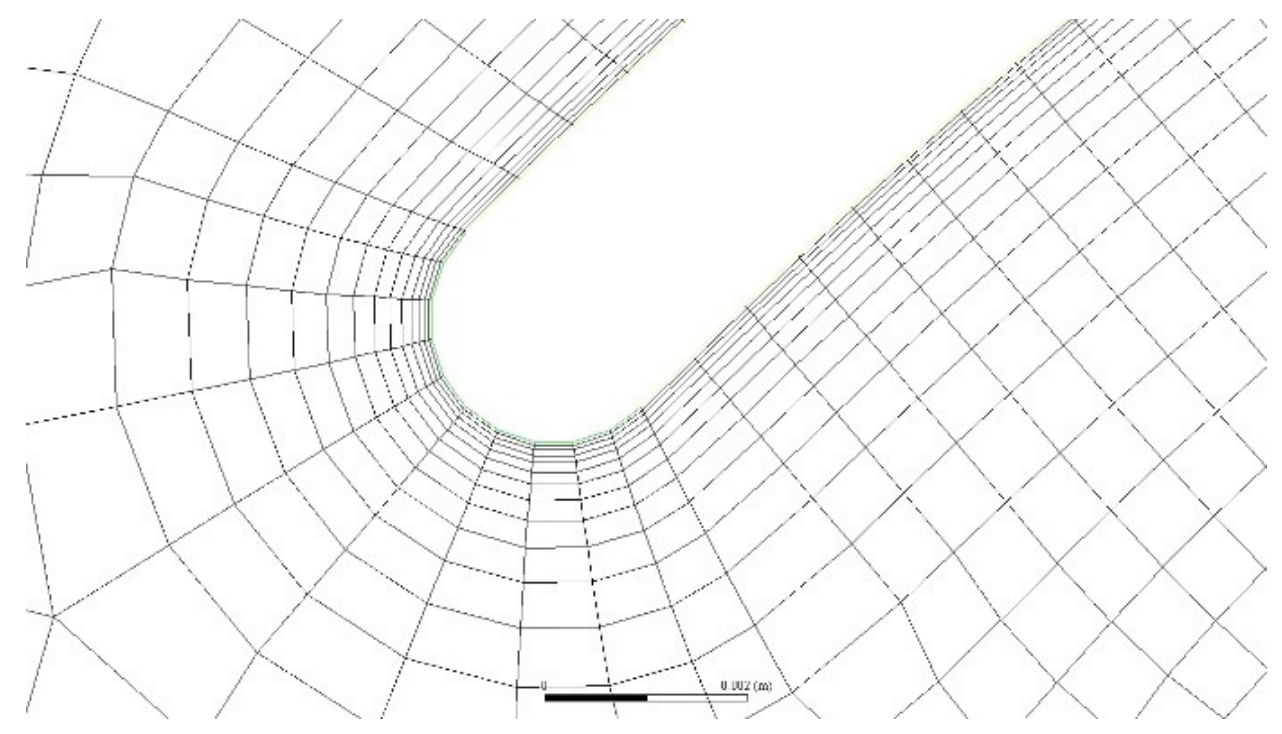

Figure 3. Meshing near the blade

The boundary conditions set for this research were the inlet, outlet, wall, and symmetry. The boundary conditions specified for the turbine blade were wall, for the inlet to set the velocity of the inlet, for the outlet to be determined for the outflow, and for the upper and lower dividers to set the symmetry. At the inlet velocity, the velocity was set at $4 \mathrm{~m} / \mathrm{s}$ [7]. The turbulent intensity value used was $1 \%[3,4]$, and the turbulent length scale was $0.08 \mathrm{~mm}$. The boundary conditions for this study are shown in Figure 1. At this stage, the selection of Rotating Zone and Stationary Zone as a mechanism for the meshing movement was also be arranged. For the Rotating Zone, the turbine blades were set to rotate with variations in angular speed proportional to the Tip Speed Ratio (TSR) variations, namely $0.2,0.5,0.7,0.9$, and 1.2 , as shown in
Figure 2

Meshing, or also known as discretization, is the division of the area in the test object model into smaller elements. These elements consist of nodes made on the specimen model as a structural boundary. These elements contain equations that will be solved numerically later. The mesh structure was made closer to the blade wall, as shown in Figure 3 .

Then after the test geometry has been created, the geometry will be simulated in the ANSYS FLUENT ${ }^{\odot}$ software. At this stage, fluid flow characteristics modeling was carried out, including selecting the solver and the turbulence model to be used. In this simulation, the k-hear Stress Transport viscous turbulence model was used [4,5]. This model was chosen to obtain accurate results, especially in 
the flow separation zone where the adverse pressure phenomenon occurs. Then, after simulating the test geometry, the data calculated was the torque coefficient and power coefficient. The coefficient of power $\left(C_{P}\right)$ and coefficient of torque $\left(C_{T}\right)$ is one of the considerations for the performance of a wind turbine. These two things show how much energy the turbine rotor can get. In theory, the $C_{P}$ value has the maximum value achieved by wind turbines. This maximum value which is also called the Betz limit has a value of $59.26 \%$. The value of this $C_{P}$ is obtained from the value of the extracted energy compared to the kinetic energy of the wind, as shown in Equation (1). The mechanical energy output from the rotor is the result of the torque generated multiplied by the rotational speed of the rotor.

$$
C_{P}=\frac{\text { extracted energy }}{\text { wind kinetic energy }}=\frac{T \omega}{\frac{1}{2} \rho A v^{3}}=C_{T} \lambda
$$

Then, the value of the torque coefficient, $C_{M}$, is the ratio or ratio between the torque value generated by the rotor and the theoretical torque of the wind. This value is obtained when the turbine is in a rotating condition, as in Equation (2).

$$
C_{M}=\frac{\text { rotor torque }}{\text { wind torque }}=\frac{T}{\frac{1}{2} \rho A v^{2} R}
$$

where $T$ is the extracted torque, $\omega$ is the rotating speed of the blade, $s$ the density of the air, $A$ is the perpendicular area of the blade, $v$ is the freestream speed, $R$ is the radius of the rotor, and $\lambda$ is the tip speed ratio.

\section{Results and Discussion}

\subsection{Comparison with The Previous Study}

A study with a simulation method produces acceptable results if a comparison has been made of the input data and the results of the simulation. Kacprzak [8,9] conducted a two-dimensional simulation of the two-bladed

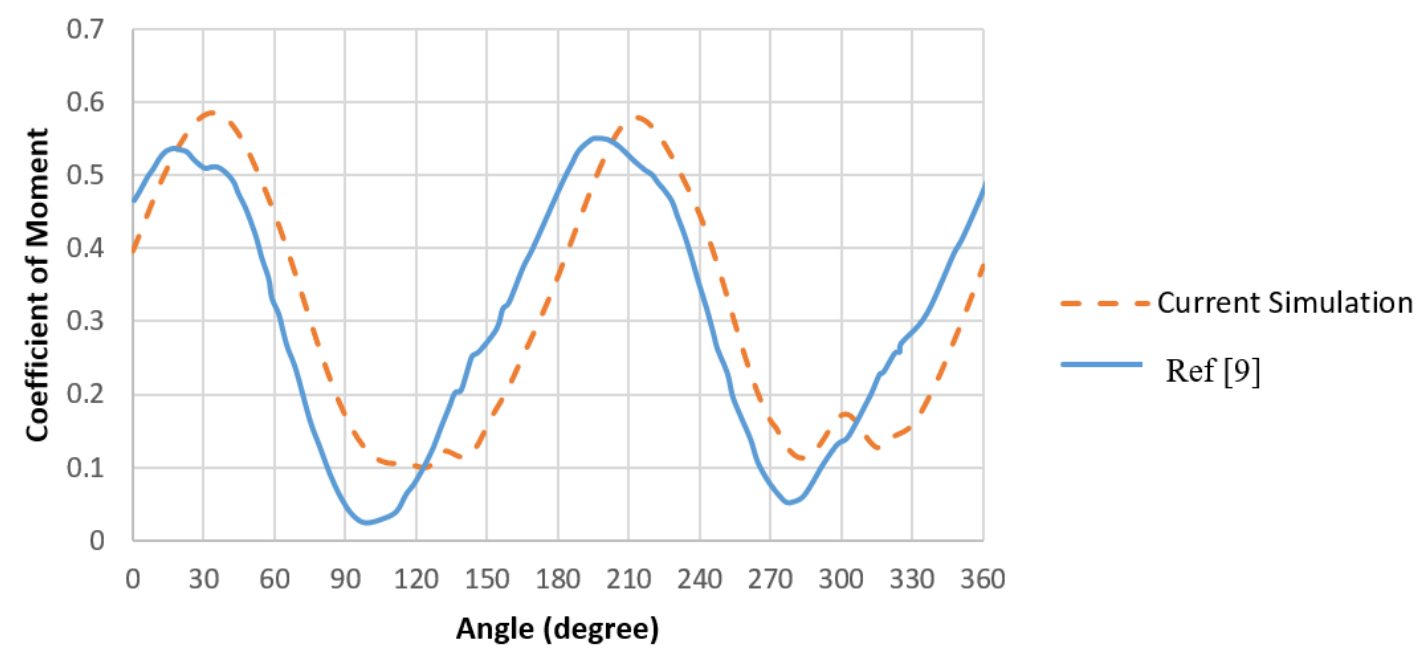

Figure 4. Comparison of moment coefficient as a function of turbine azimuth angle at $p / q=0.2$ in the current 2D Simulation and the previous study

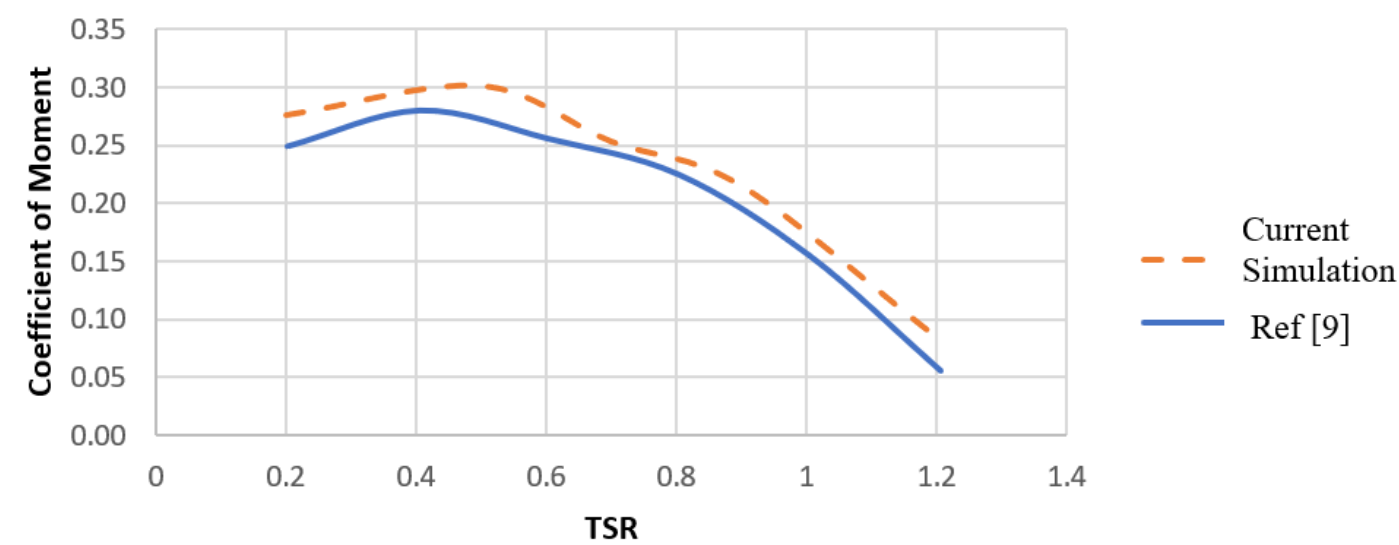

Figure 5. Comparison of moment coefficient as a function of TSR at $p / q=0.2$ to the previous study 


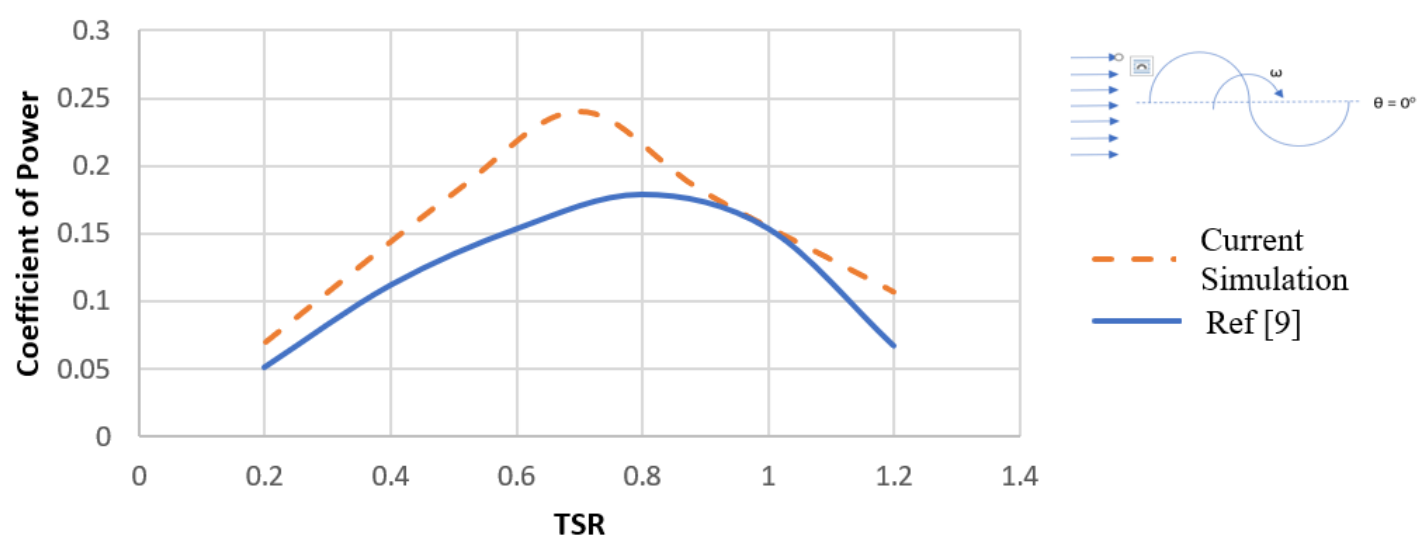

Figure 6. Comparison of power coefficient as a function of TSR to the previous study

Savonius wind turbine with a similar geometric shape, which discusses the performance comparison between the conventional Savonius turbine and the Savonius turbine, which has been modified into an elliptic shape and Bach profile blade. One of the parameters which are observed in the simulation is the blade shape factor.

In this case, the comparison method used is to examine the relevance level of the turbine rotation output from previous research to the simulation output carried out in the form of the coefficient of moment value and the coefficient of power value as well as a qualitative comparison of the flow velocity contours of the simulation results. The performance to be analyzed is the torque generated by the turbine rotation and the power coefficient of the turbine. The results of the torque value to be compared are the variations in the angle of rotation. Figure 4 shows the comparison of the torque and power coefficient values generated from this simulation and Kacprzak's simulation [9] by using the similar parameter for TSR, and quite similar geometry.

Based on Figure 4, it can be seen that the moment coefficient at each degree of rotation is different, this happens because of the geometric difference factor between the current simulation and that of [9], where the current simulation has a slightly longer diameter so that the swept area of the fluid flow in the turbine has a different value. When viewed from the calculation of the turbine power coefficient, it turns out that the current simulation results have a higher average power coefficient value compared to simulation results of [9] for the same speed of $4 \mathrm{~m} / \mathrm{s}$. Based on the graph of the comparison of the moment coefficient value against the TSR above, these two simulations have a difference in the average moment coefficient value of $9.98 \%$. The comparison of the value of the turbine power coefficient to the TSR variation can be seen in the curve in Figure6.

Based on Figure 6 above, the comparison of the coefficient of power from the current simulation with the simulation of [9], in general, the turbine performance re- sults from the current simulation show a better $C_{P}$ value. From the various TSR variations, it was found that the $C_{P}$ shown in the current simulation was higher than the simulation of [9] because of the influence of differences in geometry on the turbine diameter. This is following the theory, namely that along with the increase in the length of the center of force arm towards the center of the turbine, this increases the drag force that is generated by the system because the center point of the drag force on the advancing blade shifts to a farther radius, so that later an increase in the value of this drag is proportional to the increase in the value of the torque that works on this system which in turn leads to an increase in the resulting $C_{P}$ value. The two simulations have a difference in the value of the power coefficient of $23.27 \%$.

\subsection{Coefficient of Moment}

The moment coefficient is a dimensionless value that can help us to see the fluctuation in the value of the moment or torque that occurs at each position of the turbine rotation [8,9]. Based on the results of the simulation that has been done, the torque value for each turbine position is entered into formula 2 to calculate the moment coefficient. The distribution of the moment coefficient for one full rotation can be seen in Figures 7, 8, and 9 which show the torque cycle in the form of the maximum and minimum values for each variation of the blade shape factor displayed in the form of a sinusoidal wave.

The torque cycle that occurs in the turbine with all variations of the shape factor above shows that the peak torque character occurs alternately at several angular positions. In Figure 9, the value of the torque coefficient increases at the $30^{\circ}$ position for all TSR variations with a similar trendline between one variation and another, namely 0.75 for TSR 0.7 , followed by TSR $0.9,0.5,1.2$, and 0.2 of $0.53 ; 0.49 ; 0.44$; and 0.41 . The torque coefficient value then decreases from the angle position of $30^{\circ}$ to around $150^{\circ}$, with the minimum torque coefficient value at TSR 0.9 of 0.12 ; followed by TSR $0.5 ; 1.2 ; 0.7$; 


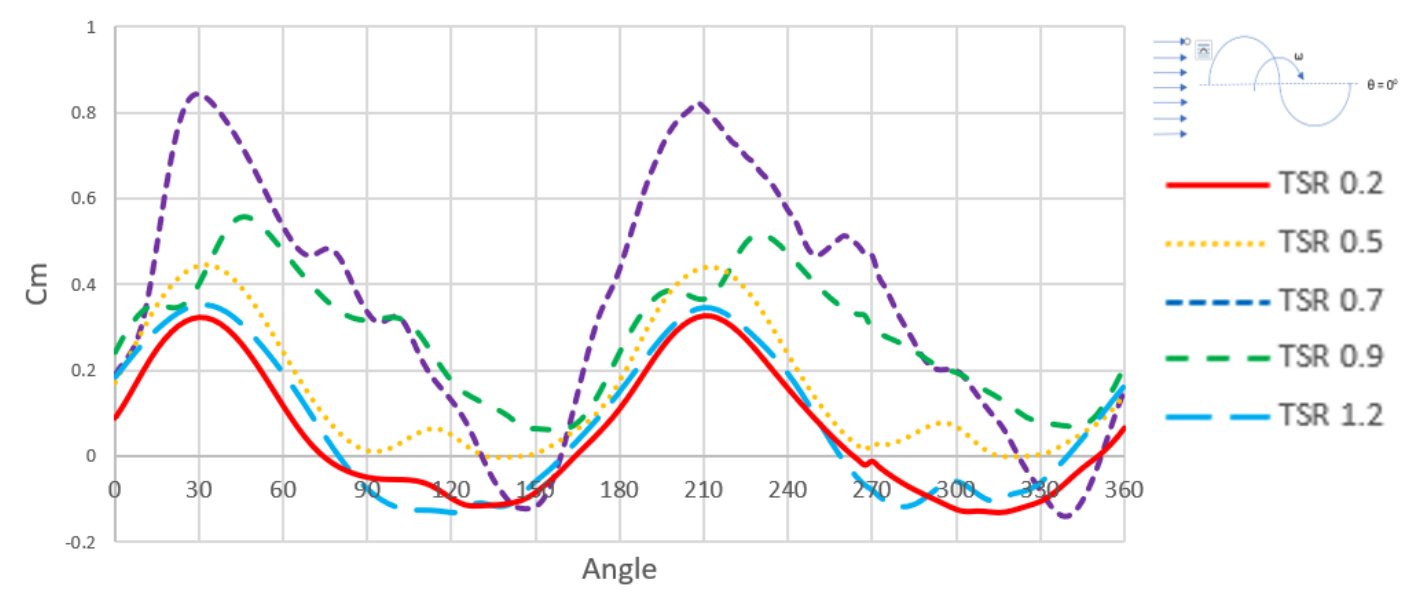

Figure 7. Savonius wind turbine torque cycle on shape factor 0.2

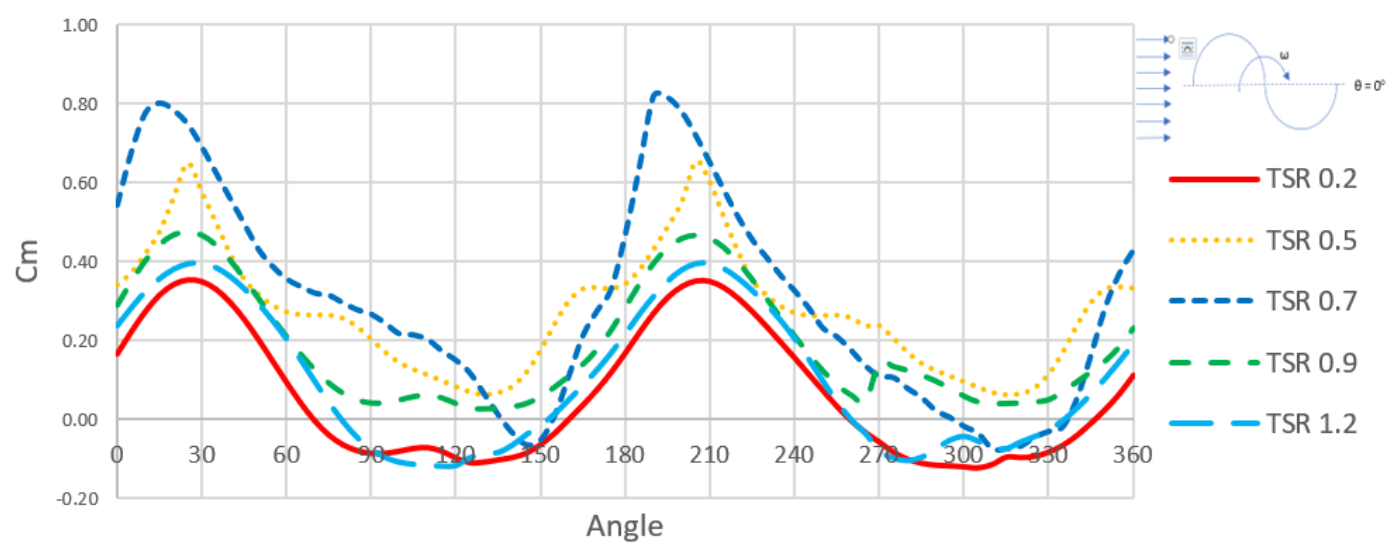

Figure 8. Savonius wind turbine torque cycle on shape factor 0.3

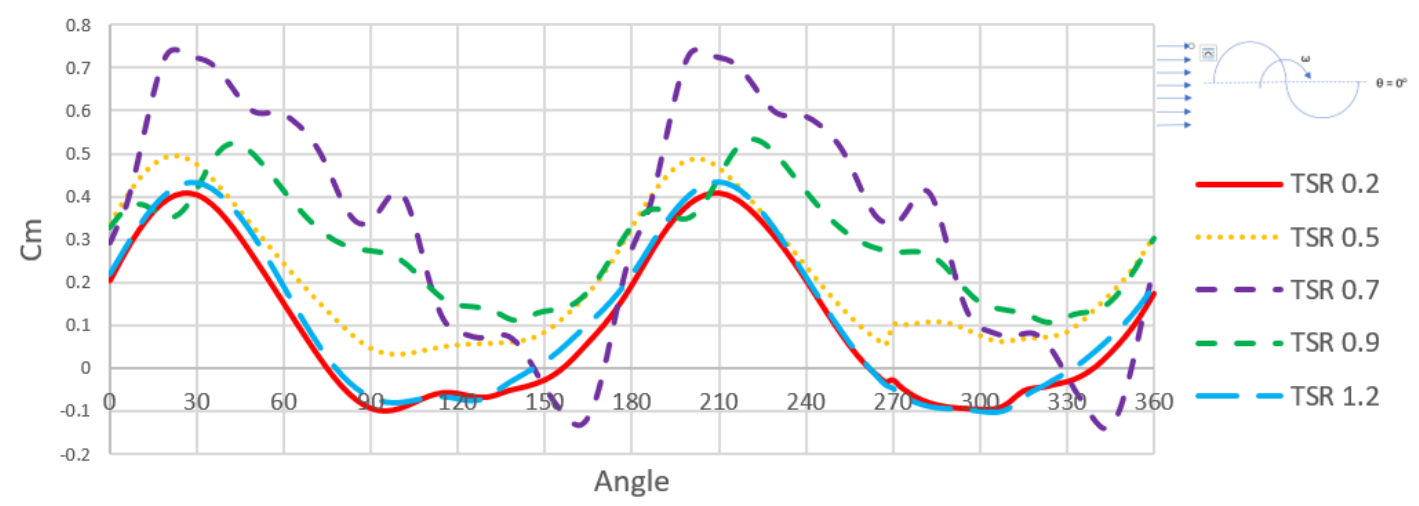

Figure 9. Savonius wind turbine torque cycle on shape factor 0.4

and 0.2 , each with a value of $0.05 ;-0.06 ;-0.86$; and 0.126 . Then the torque coefficient value increases again to the maximum value, which is when the turbine position is around $200^{\circ}$ so that the torque cycle repeats itself to the beginning.
The maximum torque of each Reynolds number value is obtained at an angle position of $30^{\circ}$, and this shows that at this angle, the turbine has the highest selfstarting value compared to other angles because the advancing blade receives a greater drag force. While the 
minimum torque is at an angular position of $150^{\circ}$ with a negative static torque value, which means that at that angle, the advancing blade receives a lower drag force from the wind than the returning blade so that external force must be applied to the blade so that it can start rotating from rest in other words. The negative value of static torque means that this variation does not have the self-starting capability [10].

In the Bach-type Savonius wind turbine run at low TSR in the current simulation, there is a negative value of static torque, starting from an angle of $65^{\circ}$ to $180^{\circ}$ with a minimum value being in an angle position of about $150^{\circ}$. A negative value indicates that at that position, the advancing blade receives lower wind drag force than the returning blade. Negative static torque means that the wind turbine does not have the ability to self-start, so that to be able to rotate from rest, it must be provided with external forces other than the force generated by the wind.

Based on Figure 10 regarding the comparison of the average moment coefficient to TSR for all variations of the shape factor above, it can be seen that the highest average moment coefficient value is obtained at TSR 0.5 for all of the shape factors. For shape factor $=0.2$, the maximum average moment coefficient value is 0.226 . As for the variations of shape factor 0.3 and 0.4 , the maximum average moment coefficient values are 0.24 and 0.257 . So it can be concluded that the shape factor 0.4 variation gives a performance increase of $6.86 \%$ compared to the variations below.

Based on the moment coefficient graph above, the results are more or less the same for all types of variations of the simulated turbine Shape factor, that is, along with the increase in the value of the varied shape factor is directly proportional to the increase in torque generated in the turbine due to the increase in the length of the center arm. The force towards the center of the turbine increases the drag force that is generated by the system because the center point of the drag force on the advancing blade is shifted to a farther radius so that the increase in the drag value is proportional to the increase in the value of the torque acting on this system.

\subsection{Flow Structure}

To analyze the simulation results in the form of a flow structure generated at a certain position, TSR $=0.7$ is used as the optimum TSR to obtain accurate comparisons of flow contours between all variations of shape factor, because the resulting power coefficient on this TSR is the largest among the other TSR.

Figure 11 shows the contours of the flow velocity of a wind turbine with a variation of the blade shape factor of $0.2 ; 0.3$; and 0.4 , which is depicted at the initial position of $0^{\circ}$, and other position that produces the highest torque, and the lowest torque. From the contour, it can be seen that the highest speed of the flow through the turbine is when it crosses the advancing blade at the rotation condition reaches an angle of $30^{\circ}$ and is also seen wake development when the turbine rotates.At the angular position $\theta=30^{\circ}$, the advancing angle experiences flow acceleration at the end, which is indicated by a reddish yellow level with a large enough area. The phenomenon of this flow acceleration can cause the creation of an elevator force that can rotate the turbine. The same phenomenon occurs periodically in advancing and returning turbine blades, which can be observed in the moment coefficient graph for each variation of the simulation run. Stagnation points also form on advancing and returning blades. At the end of the lower returning blade, there is also an increase in speed, which forms the separation point and wake area. When the angle reaches $90^{\circ}$, there is a vortex or swelling that occurs at the end of the advancing blade, which creates a downstream with a larger size. In this position, there is also a boundary layer separation which is formed near the axis of the turbine rotation. In this position, precisely in the concave part of the returning blade, there is also flow separation that occurs due to the Coanda effect which should make the flow of fluid attached to the blade surface unable to withstand the flow of fluid to remain attached to the blade. This causes separation of the concave side of the returning blade. At the angular po-

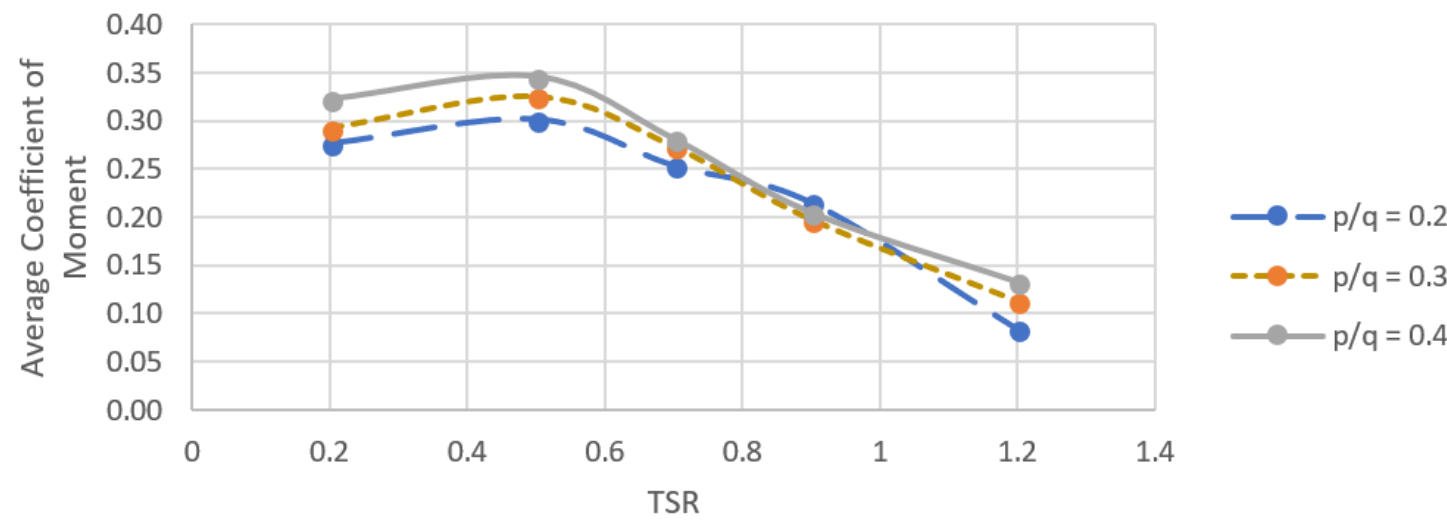

Figure 10. Comparison of average moment coefficient to TSR for all variations of the shape factor 
sition around $120^{\circ}$ to $150^{\circ}$, precisely when the torque cycle reaches the minimum torque, the stagnation point formed on the returning blade plays a role in balancing the torque that has been generated by the previous concave side advancing blade.

The difference in velocity flow contours at the above shape factor variations lies in the area of increasing velocity. At the $300^{\circ}$ position, the area with a reddish yellow color level at the tip of the advancing blade with the largest area is owned by a variation of the shape factor 0.4 , followed by a variation of 0.3 and 0.2 .

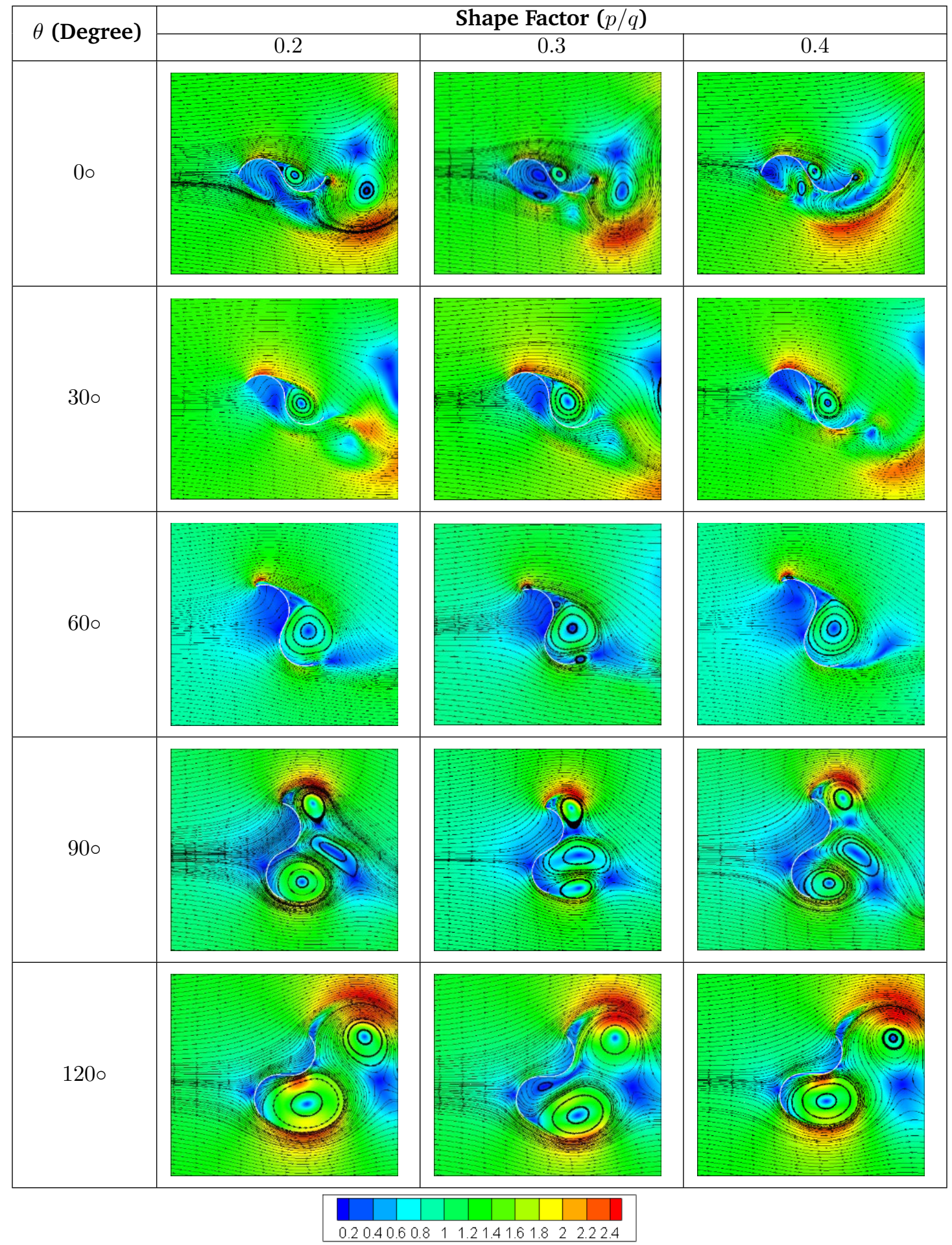

Figure 11. Dimensionless Velocity Contour $(V / V \infty)$ 


\subsection{Pressure Coefficient Distribution}

Figure 12 shows the pressure distribution along with the advancing and returning blades at turbine position of $30^{\circ}$, which results in maximum torque for all shape factors. The $\mathrm{x}$-axis on the graph shows the position on the surface (X) relative to the arc length of the blade (S). For this Bach-type Savonius turbine simulation, $X / S=0$ is at the midpoint of the front of the turbine and $X / S=1$ is at the outer end of the turbine blade.

In this Bach type Savonius turbine, the front of the advancing blade, the pressure coefficient reaches the maximum value because it collides directly with the fluid from the inlet or is the part where there is a point of stagnation. At the end of the upper advancing blade, flow acceleration occurs. This causes the pressure coefficient to drop to $C_{P}$ $=-2.3$ for the Savonius wind turbine with variations of Shape factor $0.2,-3.2$ on Shape factor 0.3 , and -4.3 on Shape factor 0.4 . On the back of the advancing blade and the back of the returning blade, the pressure coefficient value increases until the tip of the blade returns. In this area, the pressure coefficient drops slightly due to the increase in velocity at the returning blade tip. At the end of the returning blade, the pressure coefficient decreases, then the pressure coefficient value increases towards the back of the returning blade until it approaches the point of stagnation. The closer to the stagnation point, the pressure coefficient value gets closer to the value 1 .

\subsection{Coefficient of Power}

This section discusses the performance comparison of all variations of this Savonius wind turbine. This simulation uses a variation of Blade Shape factor $0.2 ; 0.3$; and 0.4 , which directly affects changes in the size of the tur- bine diameter itself. From this simulation, data is obtained in the form of the Coefficient of Power $\left(C_{P}\right)$ value as a function of the tip speed ratio (TSR) for each variation of the blade shape factor. The data obtained from the research results can be seen in Figure 13 .

Figure 13 shows the comparison of the Coefficient of Power $\left(C_{P}\right)$ of the Savonius wind turbine on all variations of the Shape factor. It can be seen that the trendline graph has a parabolic shape with the $C_{P}$ value rising to a certain TSR value, the maximum $C_{P}$ then decreasing to the maximum TSR. In the Savonius wind turbine with a Shape factor 0.2 variation, the highest $C_{P}$ value is at TSR $=0.7$ with a maximum $C_{P}$ value $=0.246$. For Shape factor 0.3 , the maximum $C_{P}$ value is 0.266 at $\mathrm{TSR}=0.7$. In simulations using a variation of Shape factor 0.4 has a maximum $C_{P}$ value of 0.281 at TSR $=0.7$. So it can be concluded that the variation of Shape factor 0.4 gives an increase in the performance of the power coefficient by $7.312 \%$ compared to the variation below.

The three $C_{P}$ graphs each show that the performance of this Bach-type Savonius wind turbine increases to a certain optimal TSR then it decreases after passing its optimal TSR. As the wind speed increases, the flow momentum hitting the advancing blade side of the turbine also increases, this allows the turbine to extract more power. Based on these data, it can be seen that the ability of the Bach-type Savonius wind turbine is not very good at low or high TSR, as evidenced by TSR 0.7 having the highest $C_{P}$ value while TSR 0.5 and 0.9 has a lower $C_{P}$ value.

Since the $C_{P}$ value of the Savonius wind turbine as a function of the Reynolds number has a parabolic graph form, it increases to the maximum $C_{P}$ at a certain Reynolds number and decrease after passing its maximum point [11,12].

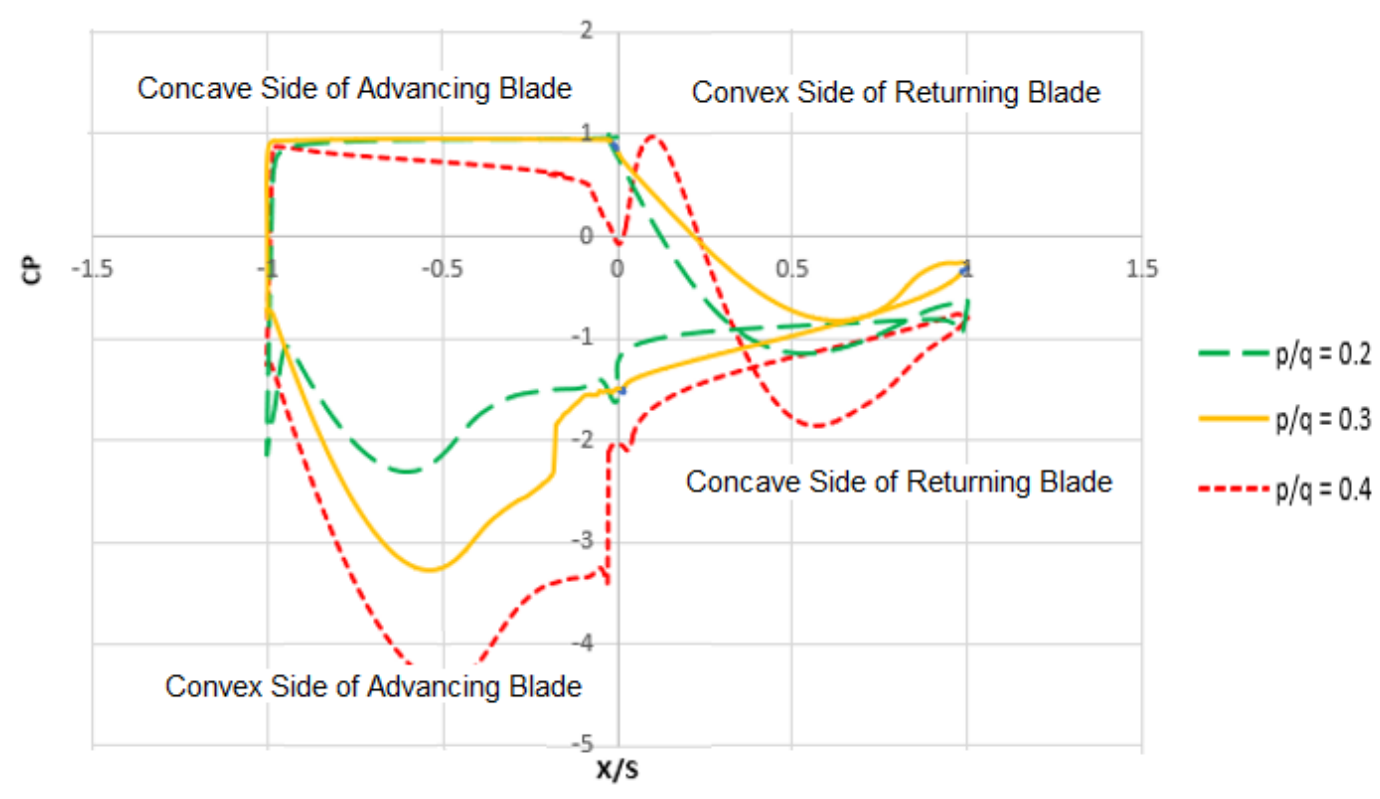

Figure 12. Pressure coefficient distribution at $\theta=30^{\circ}$ 


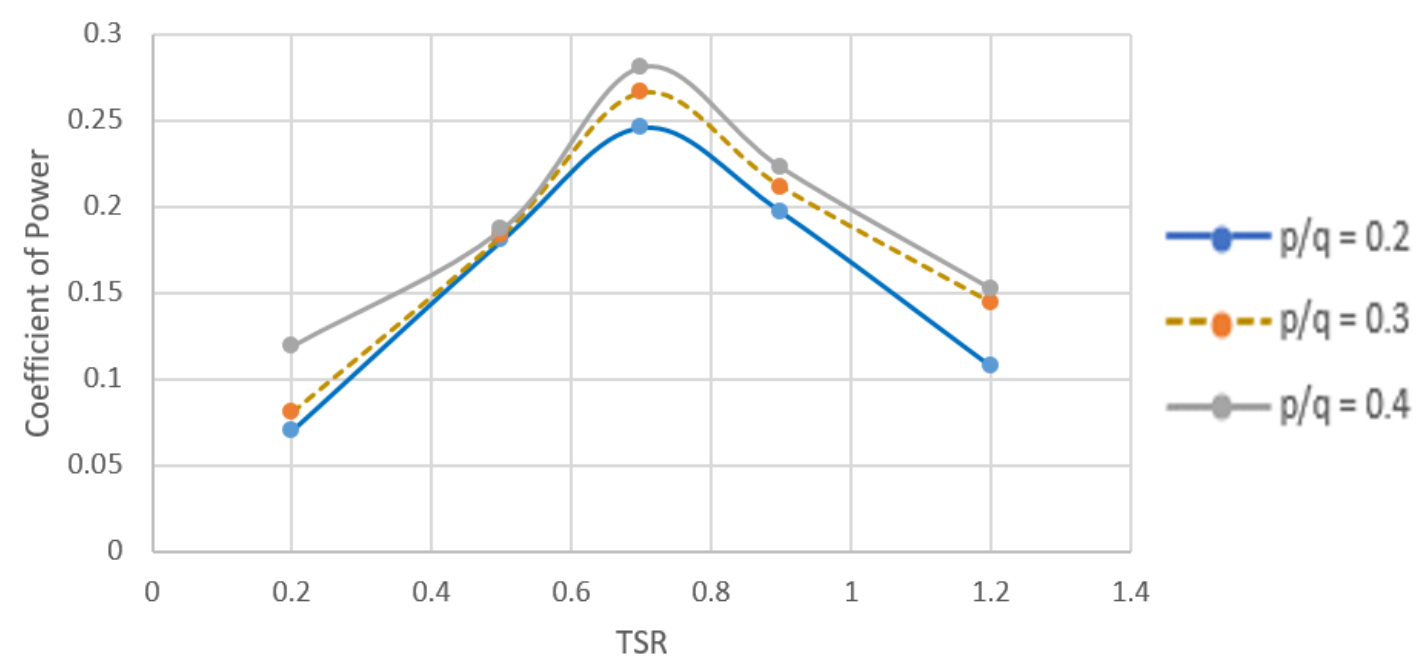

Figure 13. Comparison of the coefficient of power values for all variations of the shape factor

\section{Conclusion}

Simulation on Savonius wind turbine with Bachprofile blade has been performed. Based on the simulation results, it is found that the best performance is shown by the rotor with blade shape factor 0.4 with a maximum moment coefficient of 0.345 and 0.281 for the power coefficient, followed by the blade shape factor of 0.3 and 0.2 . Increasing the shape factor can increase the moment coefficient by $6.86 \%$ and the power coefficient by $7.31 \%$ for each variation. It can be concluded as well that the $30^{\circ}$ angle position produces the highest torque compared to other angular positions, for all variations of blade shape.

\section{References}

[1] D. Siswanto and S. Mujiyanto, Indonesia Energy Outlook. Secretariat General National Energy Council, 2019.

[2] R. Mulyana, Energi Baru Terbarukan dan Konservasi Energi 2019. Ditjen EBTKE Kementrian ESDM, 2019.

[3] J. V. Akwa, H. A. Vielmo, and A. P. Petry, "A review on the performance of Savonius wind turbines," Renewable and sustainable energy reviews, vol. 16, no. 5, pp. 3054-3064, 2012.

[4] M. Kamoji, S. B. Kedare, and S. Prabhu, "Experimental investigations on single stage modified Savonius rotor," Applied Energy, vol. 86, no. 7-8, pp. 1064 1073, 2009.

[5] A. Kumar and R. Saini, "Performance analysis of a single stage modified Savonius hydrokinetic turbine having twisted blades," Renewable Energy, vol. 113, pp. 461-478, 2017.

[6] R. Sarath Kumar, T. Micha Premkumar, S. Seralathan, and T. Mohan, "Numerical investigation of modified
Bach type vertical axis wind turbine," in Applied Mechanics and Materials, vol. 852, pp. 551-557, Trans Tech Publ, 2016.

[7] V. S. Djanali, Z. Fathurrahman, B. A. Dwiyantoro, and N. Ikhwan, "Numerical study of Savonius wind turbines with standard and Bach-profile blade variations," in AIP Conference Proceedings, vol. 2187, p. 020041, AIP Publishing LLC, 2019.

[8] K. Kacprzak, G. Liskiewicz, and K. Sobczak, "Numerical investigation of conventional and modified Savonius wind turbines," Renewable energy, vol. 60, pp. 578-585, 2013.

[9] K. Kacprzak and K. Sobczak, "Numerical analysis of the flow around the Bach-type Savonius wind turbine," in Journal of Physics: Conference Series, vol. 530, p. 012063, IOP Publishing, 2014.

[10] T. Yuwono, G. Sakti, F. N. Aulia, and A. C. Wijaya, "Improving the performance of Savonius wind turbine by installation of a circular cylinder upstream of returning turbine blade," Alexandria Engineering Journal, vol. 59, no. 6, pp. 4923-4932, 2020.

[11] H. A. H. Saeed, A. M. N. Elmekawy, and S. Z. Kassab, "Numerical study of improving Savonius turbine power coefficient by various blade shapes," Alexandria Engineering Journal, vol. 58, no. 2, pp. 429-441, 2019.

[12] N. Alom, B. Borah, and U. K. Saha, "An insight into the drag and lift characteristics of modified Bach and Benesh profiles of Savonius rotor," Energy Procedia, vol. 144, pp. 50-56, 2018. 